\title{
Procrastinación académica y bienestar psicológico en estudiantes universitarios durante las clases virtuales
}

Academic procrastination and psychological well-being in college students during virtual classes

Procrastinação acadêmica e bem-estar psicológico em universitários durante aulas virtuais

Calixto Tapullima-Mori

calixtotapullima01@gmail.com

CNL ASESORES - Perú

https://orcid.org/0000-0001-8036-2199

Gustavo Ramírez García

gustavoramirezgarcia2@gmail.com

Universidad César Vallejo-Perú

https://orcid.org/0000-0003-0035-7088

\section{RESUMEN}

La postergación de las actividades académicas ha sido por años una característica en el ámbito educativo, más aún en estudiantes universitarios, estas actitudes y comportamiento repercuten en distintos escenarios como el rendimiento, sintomatologías de ansiedad, depresión, estrés u otros, lo que se traduce en el decaimiento del bienestar, en ese sentido el estudio planteó como objetivo determinar la relación entre la procrastinación académica y bienestar psicológicos en estudiantes universitarios durante las clases virtuales. El estudio fue de tipo básico, de diseño descriptivo, correlacional y transversal, conformada por una muestra de 144 universitarios 58.3\% mujeres y $41.7 \%$ varones, con un promedio de 24.15 años y una $\mathrm{DE}=5.15$ años, aplicando la Escala de Procrastinación Académica de 12 ítems y la Escala de bienestar psicológico de 13 ítems. Se han demostrado que la procrastinación académica independiente del sexo, edad, tipo de universidad y grado de instrucción es prevalentemente medio (50\%), en tanto el bienestar psicológico también fue medio $(51.4 \%)$ con tendencia baja; de esta manera el análisis inferencial demuestra una correlación negativa a nivel general $(\mathrm{p}<.000 ;$ rho $=-.302)$ y por dimensiones en la autorregulación académica $(\mathrm{p}<.001 ;$ rho $=-.322)$ y la postergación de actividades ( $\mathrm{p}=.047$; rho=-.166) sin embargo, estos no son altamente significativos. La procrastinación se relaciona inversamente proporcional a nivel medio en la normativa de clases virtuales.

Palabras clave: Procrastinación académica. Autorregulación. Bienestar psicológico. Afecto positivo. Afecto negativo.

\section{ABSTRACT}

The postponement of academic activities has been for years a characteristic in the educational field, even more so in university students, these attitudes and behavior have an impact on different scenarios such as performance, symptoms of anxiety, depression, stress or others, which translates into the decline in well-being, in that sense the study proposed as an objective to determine the relationship between academic procrastination and psychological well-being in students. university students during virtual classes. The study was of a basic type, of descriptive, correlational and cross-sectional design, consisting of a sample of 144 university students, $58.3 \%$ women and $41.7 \%$ men, with an average of 24.15 years and a SD $=5.15$ years, applying the Academic Procrastination Scale of 12 items and the Psychological Well-being Scale of 13 items. Academic procrastination independent of sex, age, type of university and degree of education has been shown to be predominantly medium $(50 \%)$, while psychological well-being was also medium $(51.4 \%)$ with a low trend; in this way the inferential analysis demonstrates a negative correlation at the general level $(\mathrm{p}<, 000$; rho $=-.302)$ and by dimensions in academic self-regulation $(\mathrm{p}<, 001 ;$ rho $=-.322)$ and the postponement of activities $(\mathrm{p}=.047$; rho=-.166) however, these are not highly significant. Procrastination is inversely proportional to the medium level in the regulation of virtual classes.

Keywords: Academic procrastination. Self-regulation. Psychological well-being. Positive affect. Negative affect.

\section{RESUMO}

O adiamento das atividades acadêmicas tem sido há anos uma característica no campo educacional, ainda mais nos universitários, essas atitudes e comportamentos têm impacto em diferentes cenários como desempenho, sintomas de ansiedade, depressão, estresse ou outros, o que se traduz no declínio do bem-estar, nesse sentido o estudo propôs como 
objetivo determinar a relação entre procrastinação acadêmica e bem-estar psicológico nos alunos. estudantes universitários durante aulas virtuais. $\mathrm{O}$ estudo foi de um tipo básico, de design descritivo, correlacional e transversal, composto por uma amostra de 144 universitários, 58,3\% mulheres e 41,7\% homens, com média de 24,15 anos e SD = 5,15 anos, aplicando a Escala acadêmica de Procrastinação de 12 itens e a Escala de Bem-Estar Psicológico de 13 itens. A procrastinação acadêmica independente de sexo, idade, tipo de universidade e grau de escolaridade tem se mostrado predominantemente média (50\%), enquanto o bem-estar psicológico também foi médio $(51,4 \%)$ com baixa tendência; Dessa forma, a análise inferencial demonstra correlação negativa no nível geral ( $\mathrm{p}<.000$; rho=-302) e por dimensões na autorregulação acadêmica ( $\mathrm{p}<.001$; rho=-.322) e o adiamento das atividades ( $\mathrm{p}=.047$; rho=-166) no entanto, estes não são altamente significativos. A procrastinação é inversamente proporcional ao nível médio na regulação das classes virtuais.

Keywords: Academic procrastination. Self-regulation. Psychological well-being. Positive affect. Negative affect.

\section{INTRODUCCIÓN}

La pandemia a nivel mundial ha cambiado drásticamente diversos procesos sociales, dentro de las que se evidencia la educación universitaria. Como toda las demás actividades, se enfocaron en la virtualización a fin de cumplir con los objetivos y metas educacionales; su adaptación como tal ha traído consigo, diversos problemas, no solo orientados al rendimiento académico (Méndez, 2021), siendo esto el más común, sino además, los vinculados con aspectos emocionales y afectivos como el caso del bienestar psicológico (El Tiempo, 2020). Que si bien se encuentran relacionadas con el aptitud académica (Franco et al., 2019), es necesario conocer y analizar su dinámica actual.

En el contexto universitario la procrastinación se evidencia como la ausencia o desinterés del estudiante por cumplir sus actividades académicas planteadas en sus diversas asignaturas, los mismos que se han venido reforzando a través del tiempo (V. García \& Silva, 2019). Las principales características están relacionadas con el incumplimiento de sus palabras, presentación de escusas y la no atención a sus actividades académicas; por otro lado, se ha evidenciado que el aumento progresivo de éstas conductas (Ruiz \& Cuzcano, 2017), trae consigo consecuencias que alteran el bienestar psicológico del individuo, aun cuando pueda existir una diferencia entre sus tipologías (pasivas y activas).

Si bien la revisión de la bibliografía antes descrita evidencia a la procrastinación como un comportamiento disfuncional que tiene perjuicio sobre el individuo, otros autores presentan un clara diferencia entre procrastinadores pasivos y activos, siendo este último un tipo "positivo" donde presentan un mayor preferencia a trabajar bajo presión, lo que no representa una afección negativa en los resultados ya sea educativos, sociales o el desarrollo de emocional (Chu \& Choi, 2010). En Latinoamérica, tal como manifiestan diversos autores, se evidencia un alto nivel de procrastinación relacionándose con la adicción a internet, obteniendo falencias a nivel de rendimiento y el bienestar emocional o psicológico (Ramos-Galarza et al., 2017).

En el Perú la virtualización por pandemia se ha aplicado al contexto educativo, el mismo que pese a no estar en los planes encajó favorablemente para el desarrollo académico (Lovón \& Cisneros, 2020); Sin embargo, esta realidad ha reforzado en mayor medida el incumplimiento de las actividades, deberes educativos y la forma misma de la educación universitaria. Adentrando al análisis de la procrastinación académica se evidencia que existen diversos factores que están relacionados entre sí o que derivan de la mayor presencia de este, como los conflictos internos, la toma de decisiones al igual su bienestar psicológico (Ayala et al., 2020), toda vez que no puede esclarecer las medidas o estrategias a desarrollar para evitar postergar las actividades. De igual manera Vilela (2020) hace alusión a los hábitos de los estudiantes de no llevar a cabo las actividades para ser pospuestas y que estos no solo se deben considerar como una dificultad en la gestión del tiempo, sino por el contrario se tienen que abordar en mayor media con la finalidad de evitar el desarrollo de estados anímicos negativos como la ansiedad, frustración, o mala toma de decisión que a su vez se traducen en un problema para el bienestar psicológico ya sea por la complejidad de las tareas u otro factor. 
Finalmente, es importante manifestar que el estudio es importante por cuanto describe la procrastinación académica en un escenario de pandemia, donde la educación universitaria se trasladó casi en su totalidad a entornos virtuales, así se plantea como objetivo general la determinación de la relación entre la procrastinación académica y el bienestar psicológico en estudiantes universitarios.

\section{FUNDAMENTO TEÓRICO}

\section{1. antecedentes}

Para el estudio ha sido necesario analizar investigaciones previas dentro de la realidad de la Covid-19, de esta manera se inició con lo planteado por Melgaard et al. (2022) quienes manifiestan que la procrastinación académica presenta niveles considerables, de igual manera, Borger \& Morote (2021) manifiestan que los sentimientos o miedo hacia la covid-19 se relaciona significativamente con la procrastinación académica lo que implica que los niveles de bienestar no son los favorables para el afrontamiento de diversas situaciones cotidianas. Así, Yang (2021) manifiesta que la procrastinación predijo una baja satisfacción con la vida solo entre los estudiantes chinos con baja autorregulación, esta realidad supone que si bien la procrastinación acrecentó dentro del contexto mundial se evidencia diferencia a nivel de contextos geográficos.

Por otro lado (Flores \& Huamaní, 2021); Doğanülkü et al. (2021) y Hernández (2020) en sus estudios encontraron relación entre las variables de manera inversa y débil, por su parte Ganoza, (2021) evidencian una relación directa y significativa entre la procrastinación académica y el bienestar psicológico, es decir, a menor bienestar psicológico la capacidad para autorregulación y reducción de la postergación se ven disminuidos.

\subsection{Procrastinación académica}

La procrastinación académica, de un análisis de diversos autores se considera como: comportamiento del individuo que pospone de manera constante sus actividades asignadas como responsabilidad, siendo esto una evitación innecesaria que trae consigo múltiples consecuencias, además suelen justificarse por la complejidad de las tareas, su escasa motivación o el tiempo que resta para ejecutarlo. Es importante precisar que su comportamiento no solo es un problema de autocontrol y organización de tiempo (Cárdenas-Mass et al., 2020), sino además involucra factores psicosociales.

\subsection{Bienestar psicológico}

De acuerdo con la revisión de la literatura se considera como un estado emocional de satisfacción multifactorial que permite al individuo desarrollar una calidad de vida plena, caracterizada por sus componentes individuales y socioculturales que dificultan o facilitan su desarrollo (Ballesteros et al., 2006).

Adentrando al contexto educativo universitario se evidencia reducido nivel de análisis de estas variables de manera conjunta, Yarlequé et al. (2016) en su estudio con 1006 participantes de Lima y Junín se ha evidenciado que existen relación entre la procrastinación académica y el bienestar psicológico, además el nivel de procrastinación y el bienestar psicológico presentaron un nivel medio, donde no se registró diferencia estadísticamente significativas entre los estudiantes de 
Lima y Junín; por otro lado, manifestaron que los menos procrastinadores tienen mayor bienestar psicológico que los de mayor nivel de procrastinación.

\section{PROCEDIMIENTOS METODOLOGICOS}

\subsection{Tipo y diseño de investigación}

Para el desarrollo de la investigación se ha considerado un tipo de estudio básico por la naturaleza y exploración de los variables y sus componentes, el diseño de investigación fue no experimental, descriptivo, transversal y comparativo, donde los procedimientos aplicados permitieron la contrastación de la información de manera oportuna (Ato et al., 2013; Sousa et al., 2007).

\subsection{Tamaño de la muestra}

Para el estudio se consideró como población a estudiantes universitarios de la provincia de San Martín, llenaron los formularios virtuales 167 estudiantes que luego de la aplicación de una veracidad se redujo solo a una muestra de 144 universitarios (58.3\% mujeres y $41.7 \%$ varones), 24.15 en promedio con una $D E=5.15$; en relación con la información planteada se consideró un muestreo no probabilístico, por conveniencia, debido a que los estudiantes respondieron los formularios digitales de manera voluntaria (López, 2004; Otzen \& Manterola, 2017; Ventura-león, 2017).

\section{Criterios de inclusión:}

Estudiantes universitarios que cuenten con mayoría de edad

Universidades públicas o privadas

Que hayan participado de manera voluntaria

\section{Criterios de exclusión:}

Estudiantes con problemas físicos o mentales que imposibiliten responder el cuestionario

Estudiantes que abandonaron la universidad en el último mes

Aquellos que no aceptaron en consentimiento informado

\subsection{Procedimiento metodológico}

Para el desarrollo de la investigación se consideró en primer lugar la exploración bibliográfica de los instrumentos, diseñando posteriormente un formulario virtual en Google forms, con la finalidad de ser compartida a los estudiantes de pre y posgrado. Se aplicó la Escala de procrastinación académica adaptado a un contexto nacional por Domínguez et al. (2014), presentó una estructura bifactorial con 12 ítems, con escala tipo Likert que van desde $(1=$ nunca, a $5=$ siempre), el instrumento general obtuvo una confiabilidad mediante alfa de Cronbach .818, en cuanto a su dimensión autorregulación académico alcanzó un $\alpha=.821$ y para la postergación de actividades $\alpha=.752$. Centrado en un contexto local se obtuvo un $\mathrm{KMO}=.828$, a nivel general se obtuvo una confiabilidad según $\alpha=.807$ y $\Omega=.808$. A nivel general, los intervalos estuvieron planteados por bajo (12-22) medio (23-31) y alto (32-60) respectivamente, cuenta con 2 dimensiones "autorregulación académica y postergación de actividades). 
Para la evaluación del bienestar psicológico se consideró la Escala de bienestar Psicológico, adaptado por Domínguez (2014) presentó 13 ítems como una escala tipo Likert de intervalos de respuesta, en la que evidencia una estructura tetrafactorial. Logro demostrar un nivel de confiabilidad >.80; en ese sentido en el contexto local, el instrumento evidenció un KMO> .75, mientras que la confiabilidad por alfa y omega evidenciaron $\alpha=.756$ y $\Omega=.799$. Los intervalos de las puntuaciones directas estuvieron formulados a un nivel bajo (13-33), medio (34-37) y alto (38-39).

Es importante manifestar que, para el estudio, se considero el consentimiento informado antes de las respuestas mediante los formularios, siendo necesario además considerar aspectos éticos, como la confidencialidad de la información, el respeto y la no maleficencia hacia los participantes.

\subsection{Análisis estadístico}

Los datos fueron tabulados en el software estadístico del SPSS27, bajo un enfoque cuantitativo se procedió a desarrollar tablas de frecuencia, y análisis de correlación con pruebas no paramétricas, finalmente permitieron dar solución a cada uno de los objetivos planteados. La normalidad de las variables se efectuó de acuerdo con la prueba de Shapiro Willk, y las diferencias entre grupos se efectuaron mediante Kruskal-Wallis por la ausencia de normalidad y las correlaciones fueron desarrolladas mediante rho Spearman.

\section{RESULTADOS Y DISCUSIÓN}

\subsection{Resultados}

Tabla 1

Procrastinación académica en estudiantes universitarios

\begin{tabular}{|c|c|c|c|c|c|c|c|c|}
\hline & \multirow[b]{2}{*}{ f } & \multirow[b]{2}{*}{$\%$} & \multicolumn{6}{|c|}{ Diferencia $(\mathrm{p}<.05)$ Kruskal-Wallis } \\
\hline & & & Sexo & Edad & $\begin{array}{l}\text { Tipo } \\
\text { universidad }\end{array}$ & de & $\begin{array}{l}\text { Nivel } \\
\text { instrucción }\end{array}$ & de \\
\hline Bajo & 45 & 31.3 & \multirow{4}{*}{.146} & \multirow{4}{*}{.064} & \multirow{4}{*}{\multicolumn{2}{|c|}{.541}} & \multirow{4}{*}{.220} & \\
\hline Medio & 72 & 50.0 & & & & & & \\
\hline Alto & 27 & 18.8 & & & & & & \\
\hline Total & 144 & 100.0 & & & & & & \\
\hline
\end{tabular}

Nota: medidas percentilares 25, $75($ Bajo $=<22 ;$ medio $=23-31 ;$ alto $=>32)$

El estudio descriptivo evidenció que la procrastinación académica en estudiantes universitarios es prevalentemente de nivel medio (50\%) con tendencia a bajo $(31.3 \%)$, además muestra que, de acuerdo con el sexo, es más prevalente en varones (63\%) que en mujeres (37\%); comprendidos entre 21-27 años (55.6\%); de universidades privadas (55.6\%) en pregrado (100\%). Es preciso manifestar además que no se ha demostrado diferencia significativa de las medias alcanzadas de los participantes mediante Kruskal-Wallis por cuanto el valor p fue mayor a .050 en cada uno de los factores como sexo, edad, tipo de universidad y el nivel de instrucción. 
Tabla 2

Bienestar psicológico

\begin{tabular}{lllllll}
\hline & & \multicolumn{5}{l}{ Diferencia $(\mathbf{p}<.05)$ Kruskal-Wallis } \\
\cline { 5 - 7 } & $\mathbf{f}$ & $\%$ & Sexo & Edad & $\begin{array}{l}\text { Tipo de } \\
\text { universidad }\end{array}$ & $\begin{array}{l}\text { Nivel de } \\
\text { instrucción }\end{array}$ \\
\hline Bajo & 45 & 31.3 & & & & \\
Medio & 74 & 51.4 & .957 & .314 & .158 & .934 \\
Alto & 25 & 17.4 & & & & \\
Total & 144 & 100.0 & & & & \\
\hline
\end{tabular}

Nota: medidas percentilares 25,75 (bajo $=<33$; medio $=34-37$; alto $=>38$ )

De acuerdo con la evaluación de los datos se han observado que existe prevalencia media de bienestar psicológico (51.4\%), con una tendencia a bajo $(31.5 \%)$, el mismo que evidencia un manejo de situaciones menos aprovechables, al igual que un vínculo social ciertamente inestable. De igual manera el bienestar psicológico es alto en mujeres en mayor medida que en los varones en edades comprendidas entre los 21-27 años de las universidades privadas que cursan el pregrado. No obstante, de acuerdo con el estadístico Kruskal-Wallis pese a las diferencias de las medias, estas no han sido estadísticamente significativas $(\mathrm{p}<.050)$ en relación con el sexo, edad, tipo de universidad y nivel de instrucción.

Tabla 3

Correlación entre procrastinación académica y bienestar psicológico

\begin{tabular}{lcccc}
\hline & Shapiro-Willk & \multicolumn{2}{c}{ Bienestar psicológico } & \\
& $\mathbf{p}$ & $\mathbf{N}$ & $\boldsymbol{r h o}$ & $\mathbf{p}$ \\
\hline Procrastinación académica & .308 & & -.303 & $<.000$ \\
-Autorregulación académica & .079 & 144 & -.322 & $<.000$ \\
-Postergación de actividades & .007 & & -.166 & .047 \\
\hline
\end{tabular}

Nota: relación estadísticamente significativa a .001

La tabla evidencia que, para el desarrollo de la contrastación estadística, fue necesario desarrollar la prueba de normalidad mediante Shapiro Willk por ser de mayor eficiencia (Dagnino, 2014; Pedrosa et al., 2015), obteniendo de esta manera una significancia <.050; razón por la que determinó el uso de pruebas no paramétricas como Rho de spearman; los resultados demuestran que existe relación inversamente proporcional a nivel de la variable general "procrastinación académica" con el bienestar psicológico ( $\mathrm{p}<.000$; rho=-.303) de igual manera las dimensiones están relacionadas de manera significativa. 
Tabla 4

Correlaciones de acuerdo con los datos descriptivos

\begin{tabular}{|c|c|c|c|c|}
\hline & & \multicolumn{3}{|c|}{ Bienestar psicológico } \\
\hline & & $\mathbf{n}$ & rho & $\mathbf{p}$ \\
\hline \multirow{3}{*}{ Edad } & $18-20$ años & 40 & -.493 & .001 \\
\hline & 21-27 años & 72 & -.359 & .002 \\
\hline & 28-47 años & 32 & -.233 & .194 \\
\hline \multirow{2}{*}{ Sexo } & Mujer & 84 & -.256 & .019 \\
\hline & Varón & 60 & -.362 & .004 \\
\hline \multirow{2}{*}{ Universidad } & Pública & 53 & -.528 & .000 \\
\hline & Privada & 91 & -.189 & .079 \\
\hline \multirow{2}{*}{ Grado académico } & Pregrado & 130 & -.278 & .001 \\
\hline & Posgrado & 14 & -.627 & .016 \\
\hline
\end{tabular}

Nota: Segmentación de los datos, $\mathrm{p}<.001$

Se ha evidenciado que de acuerdo con los criterios descriptivos ha existido relación inversamente proporcional y significativa (edad, sexo, universidad, grado académico) no obstante en participantes entre los 27-47 años de universidades privadas no se han demostrado la relación estadísticamente significativa ( $\mathrm{p}>.005)$.

\subsection{Discusión}

La aplicación de la escala de Procrastinación Académica obtuvo como resultado un nivel predominantemente medio del 50\%, resultados similares fueron presentados por Porras y Ortega (2021) que alcanzaron un nivel medio de procrastinación, por su parte Aznar-Díaz et al. (2020) estimaron la procrastinación en un rango por encima del 50\%, además los resultados no evidencian un diferencia entre los factores demográficos como sexo, edad, tipo de universidad o nivel de instrucción, demostrando así que la procrastinación está presente en similares proporciones, sin embargo, Rodríguez \& Clariana (2017) evidencian resultado un tanto similares con 105 estudiantes universitarios, donde la procrastinación depende de la edad y no del nivel en que estos se encuentran, en contraste con el análisis estadístico mediante Kruskal-Wallis la edad, tiende a acercarse en mayor medida a una diferencia significativa con un valor $\mathrm{p}=.064$, de igual manera no existe diferencia estadística entre los encuestados masculinos y femeninos para el comportamiento de procrastinación (Rahoo et al., 2021); para mejorar estos escenarios es necesario el desarrollo de diversas actividades de manera articulada, con la integración de la comunidad universitaria, así resulta necesario plantear el uso de terapias para su abordaje oportuno y alcanzar resultados que favorezcan a la población universitaria, tal como manifiesta Kian et al. (2020) quienes luego de aplicar la terapia de aceptación obtuvieron una mejora estadísticamente significativa $(\mathrm{p}<.005)$ en la reducción de la procrastinación, de igual manera se puede repotenciar las habilidades de planificación y organización (Bison et al., 2021); a todo ello es importante considerar las consecuencias que la procrastinación académica representa en la comunidad universitaria. 
Los resultados de la aplicación de la escala bienestar psicológico demostraron que los estudiantes universitarios alcanzaron un nivel medio $51.4 \%$ en el contexto de educación remota, guardando relación con el $71.9 \%$ de bienestar alto y $25.4 \%$ moderado obtenido por Ruiz et al. (2020), de igual manera obtienen que los factores de sexo, edad o grado académico no se diferencian, estos resultados similares a los datos obtenidos mediante la prueba de Kruskal-Wallis ( $p>.05$ ), así los factores descriptivos no han tenido una diferencia a consecuencia de la educación universitaria a distancia, por otro lado también es preciso manifestar que dentro de este escenario los niveles de bienestar psicológico no se han visto afectado por el desarrollo de clases universitarias de manera virtual (Araque-Castellanos et al., 2020), además en contraste con los resultados pese a la existencia de diferencia a nivel sexo se ha registrado que las mujeres presentan mejores niveles de bienestar en comparación que los hombres. Por otro lado, si bien el bienestar psicológico es una necesidad para garantizar aspectos relacionados con la calidad de vida, su mejora puede ser implementada haciendo uso de diversos recursos como los programas de prevención universitarios (Küchler et al., 2019; Rabin et al., 2011; Silvera-Torres et al., 2020), de igual manera la contribución docente-universidad tiene que involucrar un mejor control sin establecer diferencia de los niveles académicos, pues el bienestar psicológico es de importancia para todos.

En un análisis de la correlación a nivel general fue necesario aplicar la prueba de normalidad mediante Shapiro Willk por su robustez, en la que se evidenció una población no distribuida con normalidad, que dio paso a la aplicación de una prueba no paramétrica, demostrando que la procrastinación académica guarda relación directa y negativa $(\mathrm{p}<.050$; rho=-.303), teóricamente explicado, debido a que cuando mayor son los niveles de procrastinación menor serán los niveles de bienestar alcanzado en los estudiantes universitarios. En cuanto a sus dimensiones, la autorregulación ha presentado un mayor nivel de correlación siendo esto necesario para el desarrollo de competencias educativas (M. García, 2012); los datos descritos anteriormente guardan relación con lo manifestado por Yarlequé et al. (2016) quienes en su estudio de 1006 participantes de Lima y Junín evidencian que existen relación entre la procrastinación académica y el bienestar psicológico y que no existe diferencia estadísticamente entre los factores descriptivos, finalmente se han encontrado que los niveles de correlación directa y negativa ha estado presente en mayor medida en estudiantes de posgrado (rho=-.627) pertenecientes a universidades públicas $(\mathrm{rho}=-.528)$, lo que demuestra la relevancia del estudio para reforzar el conocimiento acerca de la importancia que tiene el estudio de la procrastinación y en qué medida éstas tienen una repercusión en el bienestar psicológico; en tanto también se han demostrado que pese a un entorno de estudio nuevo como lo son las plataformas virtuales no ha tenido injerencia en el cambio de la procrastinación en contraste con los estudios antes analizados.

\section{CONCLUSIÓN}

Se ha evidenciado que el $50 \%$ de los estudiantes universitarios que desarrollan sus clases de manera virtual alcanzaron un nivel medio de procrastinación, de igual manera el bienestar psicológico fue medio (51.4\%) con tendencia baja; lo que implica que en cierto modo el estudiante no se logra adaptar considerablemente a las nuevas exigencias que implica las clases remoto. El análisis inferencial demuestra una correlación negativa a nivel general y por dimensiones, sin embargo, estos no son altamente significativos. Dentro de los datos relevantes se evidencian que a nivel de posgrado la correlación negativa es más alta, debido a que estos tienden a desarrollar menor índice de procrastinación ( $\mathrm{p}<.016$; rho=-.627); similares datos se presentan en estudiantes de universidades públicas. El presente estudio identificó limitaciones metodológicas, por cuanto el tamaño de muestra no alcanzó un nivel de representatividad esperada, además el método del muestreo fue no probabilístico. Teóricamente, la relación entre estas variables es recientemente investigada, además de contar con una variable constante como es la Covid-19, es así que, para estudios futuros se establezcan análisis longitudinales y bibliométricos para comparar los niveles de procrastinación antes y durante la educación en línea. 


\section{REFERENCIAS}

Araque-Castellanos, F., González-Gutiérrez, O., López-Jaimes, R. J., Nuván-Hurtado, I. L., \& Medina-Ortiz, O. (2020). Psychological well-being and sociodemographic characteristics in university students during quarantine by SARS-COV-2. Archivos Venezolanos de Farmacologia y Terapeutica, 39(8), 998-1004. https://doi.org/10.5281/zenodo.4544052

Ato, M., Juan, L., \& Benavente, A. (2013). Un sistema de clasificación de los diseños de investigación en psicología. Anales de Psicologia, 29(3), 1038-1059. https://doi.org/10.6018/analesps.29.3.178511

Ayala, A. S., Rodríguez, R. Y., Villanueva, W., \& Campos, M. (2020). La procrastinación académica : teorías, elementos y modelos. Muro de la Investigación, 2, 40-52. https://doi.org/10.17162/rmi.v5i2.1324

Aznar-Díaz, I., Romero-Rodríguez, J. M., García-González, A., \& Ramírez-Montoya, M. S. (2020). Mexican and Spanish university students' Internet addiction and academic procrastination: Correlation and potential factors. PLoS ONE, 15(5), 1-18. https://doi.org/10.1371/journal.pone.0233655

Ballesteros, B. P., Medina, A., \& Caycedo, C. (2006). El bienestar psicológico definido por asistentes a un servicio de consulta psicológica en Bogotá, Colombia. Universitas psychologica, 5(2), 239-258. http://www.scielo.org.co/scielo.php?script=sci_arttext\&pid=S1657-92672006000200004

Bison, I., Bison, I., Civilleri, A., \& Fraccaroli, F. (2021). Ritardo negli studi accademici: il ruolo della procrastinazione irrazionale e delle capacità di pianificazione ed or- ganizzazione dello studio. Giornale italiano di psicologia, XLVIII(1/2021), 273-283. https://doi.org/10.1421/101242

Borger, N., \& Morote, F. S. (2021). Procrastinación académica y bienestar psicológico en estudiantes universitarios [Universidad Católica de Santa María]. En Universidad Católica de Santa María. https://tesis.ucsm.edu.pe/repositorio/handle/UCSM/10687

Cárdenas-Mass, P. M., Hernández-Marín, G. del J., \& Cajigal, E. (2020). Procrastinación Académica en Estudiantes Universitarios de una Institución Pública : Caso de la Facultad de Ciencias Educativas. Revista RedCA, 3(9), 1840.

Chu, A. H. C., \& Choi, J. N. (2010). Rethinking Procrastination: Positive Effects of “Active” Procrastination Behavior on Attitudes and Performance. The Journal of Social Psychology, 145(3), 245-264. https://doi.org/10.3200/SOCP.145.3.245-264

Dagnino, J. (2014). La distribución normal. Rev Chil Anest, 43, 116-121. http://www.scielo.org.co/pdf/rups/v14n1/v14n1a21.pdf

Doğanülkü, H. A., Korkmaz, O., Griffiths, M. D., \& Pakpour, A. H. (2021). Fear of COVID-19 lead to procrastination among Turkish university students: The mediating role of intolerance of uncertainty. BMC Psychology, 9(1), 1-8. https://doi.org/10.1186/S40359-021-00681-9/TABLES/2

Dominguez, A. S. (2014). Análisis psicométrico de la escala de bienestar psicológico para adultos en estudiantes universitarios de lima: Un enfoque de ecuaciones estructurales. Psychologia: avances de la disciplina, 8(1), 2331. http://www.scielo.org.co/pdf/psych/v8n1/v8n1a03.pdf

Dominguez, S. A., Villegas, G., \& Centeno, S. B. (2014). Procrastinación Académica: Validación de una escala en una muestra de estudiantes de una universidad privada. Liberabit, 20(2), 293-304. http://www.scielo.org.pe/pdf/liber/v20n2/a10v20n2.pdf

El Tiempo. (2020, mayo 11). Salud mental, clases virtuales y educación superior. El tiempo. https://www.eltiempo.com/vida/educacion/salud-mental-clases-virtuales-y-educacion-superior-494212

Flores, L., \& Huamaní, P. (2021). Procrastinación académica y bienestar psicológico en estudiantes de economía de la Universidad Nacional de Huancavelica - 2020 [Universidad Peruana Los Andes]. En Universidad Peruana Los Andes. http://repositorio.upla.edu.pe/handle/20.500.12848/2377

Franco, J. F., Osorio, A. V., \& Cervantes, X. P. (2019). Relación entre el bienestar psicológico, rendimiento académico y acoso en los estudiantes universitarios. Universidad y Sociedad, 11(5), 301-308. http://scielo.sld.cu/pdf/rus/v11n1/2218-3620-rus-11-01-265.pdf

Ganoza, L. M. B. (2021). Procrastinación académica y bienestar psicológico en estudiantes de especialidades psicopedagógicas de una institución privada de Lima [Universidad Privada Antenor Orrego]. En Universidad Privada Antenor Orrego. https://repositorio.upao.edu.pe/handle/20.500.12759/8307

García, M. (2012). La autorregulación académica como variable explicativa del los procesos de aprendizaje universitario. Profesorado. Revista de Currículum y Formación de Profesorado, 16(1), 203-221. https://www.redalyc.org/articulo.oa?id=56724377012

García, V., \& Silva, P. M. (2019). Procrastinación académica entre estudiantes de cursos en línea. Validación de un cuestionario. Apertura (Guadalajara, Jal.), 11(2), 122-137. https://doi.org/10.32870/AP.V11N2.1673

Hernández, N. M. (2020). Procrastinación académica, estrés académico y bienestar psicológico en estudiantes de psicología de una universidad privada de Chincha, 2020 [Universidad de San Martín de Porres]. En Repositorio 
Académico USMP. https://repositorio.usmp.edu.pe/handle/20.500.12727/7822?show=full

Kian, A. A., Rostami, B., Moosavi, S. E., Maghbooli, M., \& Fakoor, E. (2020). The effectiveness of acceptance and commitment therapy on academic procrastination in medical sciences students of Zanjan University. Iranian Journal of Psychiatry and Clinical Psychology, 26(2), 142-153. https://doi.org/10.32598/ijpcp.26.2.2817.1

Küchler, A. M., Albus, P., Ebert, D. D., \& Baumeister, H. (2019). Effectiveness of an internet-based intervention for procrastination in college students (StudiCare Procrastination): Study protocol of a randomized controlled trial. Internet Interventions, 17, 100245. https://doi.org/10.1016/J.INVENT.2019.100245

López, P. (2004). Población muestra y muestreo. Punto Cero, 9(8), 69-74. http://www.scielo.org.bo/pdf/rpc/v09n08/v09n08a12.pdf

Lovón, M. A., \& Cisneros, S. A. (2020). Repercusiones de las clases virtuales en los estudiantes universitarios en el contexto de la cuarentena por COVID-19: El caso de la PUCP. Propósitos y Representaciones, 8(SPE3), e588. https://doi.org/10.20511/pyr2020.v8nspe3.588

Melgaard, J., Monir, R., Lasrado, L. A., \& Fagerstrøm, A. (2022). Academic Procrastination and Online Learning During the COVID-19 Pandemic. Procedia computer science, 196, 117-124. https://doi.org/10.1016/J.PROCS.2021.11.080

Méndez, C. R. (2021). Procrastinación e incremento del estrés en docentes y estudiantes universitarios frente a la educación online. Revista Scientific, 6(20), 62-78. https://doi.org/10.29394/SCIENTIFIC.ISSN.25422987.2021.6.20.3.62-78

Otzen, T., \& Manterola, C. (2017). Técnicas de Muestreo sobre una Población a Estudio. International Journal of Morphology, 35(1), 227-232. https://doi.org/10.4067/S0717-95022017000100037

Pedrosa, I., Juarros-Basterretxea, J., Robles-Fernández, A., Basteiro, J., \& García-Cueto, E. (2015). Pruebas de bondad de ajuste en distribuciones simétricas, ¿qué estadístico utilizar? Universitas Psychologica, 14(1), 245-254. https://doi.org/10.11144/Javeriana.upsy13-5.pbad

Porras, M. M., \& Ortega, F. H. (2021). Procrastination, test anxiety and academic performance on university students. Interdisciplinaria, 38(2), 243-258. https://doi.org/10.16888/INTERD.2021.38.2.16

Rabin, L. A., Fogel, J., \& Nutter-Upham, K. E. (2011). Academic procrastination in college students: the role of selfreported executive function. Journal of clinical and experimental neuropsychology, 33(3), 344-357. https://doi.org/10.1080/13803395.2010.518597

Rahoo, L. A., Abbasi, M. S., \& Jatiyal, A. A. (2021). The Role of Social Networks in Delay of University Students Academics in Pakistan. Library Philosophy and Practice, 4792. https://digitalcommons.unl.edu/libphilprac/4792/

Ramos-Galarza, C., Jadán-Guerrero, J., Paredes-Núñez, L., Bolaños-Pasquel, M., \& Gómez-García, A. (2017). Procastination, internet addiction, and academic performance in Ecuadorian college students. Estudios pedagógicos (Valdivia), 43(3), 275-289. https://doi.org/10.4067/S0718-07052017000300016

Rodríguez, A., \& Clariana, M. (2017). Procrastinación en Estudiantes Universitarios: Su relación con la edad y el curso académico. Revista Colombiana de Psicologia, 26(1), 45-60. https://doi.org/10.15446/RCP.V26N1.53572

Ruiz, C., \& Cuzcano, A. (2017). El Estudiante Procrastinador. Revista EDUCA UMCH, 9, 23-33. https://doi.org/10.35756/educaumch.v9i0.30

Ruíz, L. C., Blanco-González, L., Araque-Castellanos, F., Mendoza-Rincón, B. M., \& Arenas-Villamizar, V. V. (2020). Características del bienestar psicológico en jóvenes universitarios en el marco aislamiento preventivo por COVID-19. Gaceta Medica de Caracas, 128(Supl 2), S359-S364. https://doi.org/10.47307/GMC.2020.128.S2.22

Silvera-Torres, L., Olivella-López, G., Cudris-Torres, L., Bahamón, M., \& Gil-Ángel, G. (2020). Programas, estrategias y protocolos de bienestar psicológico implementados en jóvenes universitarios. Archivos Venezolanos de Farmacología y Terapéutica, 39(3), 322-325. https://doi.org/10.5281/zenodo.4069559

Sousa, V. D., Driessnack, M., \& Costa, I. A. (2007). Revisión de diseños de investigación resaltantes para enfermería. parte 1: Diseños de investigación cuantitativa. Revista Latino-am Enfermagem, 15(3). https://www.scielo.br/pdf/rlae/v15n3/es_v15n3a22.pdf

Ventura-león, J. L. (2017). ¿Población o muestra?: Una diferencia necesaria. Revista Cubana de Salud Pública, 43(3), 648-649. http://scielo.sld.cu/pdf/rcsp/v43n4/spu14417.pdf

Vilela, P. (2020, junio 3). La procrastinación en los tiempos de la educación virtual y el teletrabajo. Pontificia Universidad Católica del Perú. https://puntoedu.pucp.edu.pe/voces-pucp/la-procrastinacion-en-los-tiempos-de-laeducacion-virtual-y-el-teletrabajo/

Yang, Z. (2021). Does Procrastination Always Predict Lower Life Satisfaction? A Study on the Moderation Effect of Self-Regulation in China and the United Kingdom. Frontiers in Psychology, 12, 2645. https://doi.org/10.3389/FPSYG.2021.690838/BIBTEX

Yarlequé, L. A., Javier, L., Monroe, J. D., Nuñez, E. R., Navarro, L. L., Padilla, M., Matalinares, M. L., Navarro, L. R., \& Campos, J. (2016). Procrastinación, estrés y bienestar psicológico en estudiantes de educación superior de Lima y Junín. Horizonte de la Ciencia, 6(10), 184. https://doi.org/10.26490/UNCP.HORIZONTECIENCIA.2016.10.213 